\title{
Generic estimator of biomass concentration for Escherichia coli and Saccharomyces cerevisiae fed-batch cultures based on cumulative oxygen consumption rate
}

\author{
Renaldas Urniezius ${ }^{1 *} \mathbb{1}$, Arnas Survyla ${ }^{1}$, Dziugas Paulauskas² Vladas Algirdas Bumelis $^{2}$ \\ and Vytautas Galvanauskas ${ }^{1}$
}

\begin{abstract}
Background: The focus of this study is online estimation of biomass concentration in fed-batch cultures. It describes a bioengineering software solution, which is explored for Escherichia coli and Saccharomyces cerevisiae fed-batch cultures. The experimental investigation of both cultures presents experimental validation results since the start of the bioprocess, i.e. since the injection of inoculant solution into bioreactor. In total, four strains were analyzed, and 21 experiments were performed under varying bioprocess conditions, out of which 7 experiments were carried out with dosed substrate feeding. Development of the microorganisms' culture invariant generic estimator of biomass concentration was the main goal of this research.

Results: The results show that stoichiometric parameters provide acceptable knowledge on the state of biomass concentrations during the whole cultivation process, including the exponential growth phase of both E. coli and S. cerevisiae cultures. The cell culture stoichiometric parameters are estimated by a procedure based on the Luedeking/ Piret-model and maximization of entropy. The main input signal of the approach is cumulative oxygen uptake rate at fed-batch cultivation processes. The developed noninvasive biomass estimation procedure was intentionally made to not depend on the selection of corresponding bioprocess/bioreactor parameters.
\end{abstract}

Conclusions: The precision errors, since the bioprocess start, when inoculant was injected to a bioreactor, confirmed that the approach is relevant for online biomass state estimation. This included the lag and exponential growth phases for both E. coli and S. cerevisiae. The suggested estimation procedure is identical for both cultures. This approach improves the precision achieved by other authors without compromising the simplicity of the implementation. Moreover, the suggested approach is a candidate method to be the microorganisms' culture invariant approach. It does not depend on any numeric initial optimization conditions, it does not require any of bioreactor parameters. No numeric stability issues of convergence occurred during multiple performance tests. All this makes this approach a potential candidate for industrial tasks with adaptive feeding control or automatic inoculations when substrate feeding profile and bioreactor parameters are not provided.

Keywords: Biomass estimator, Stoichiometry, Relative entropy, Microbial cultivation

\footnotetext{
*Correspondence: renaldas.urniezius@ktu.lt

${ }^{1}$ Department of Automation, Kaunas University of Technology,

51367 Kaunas, Lithuania

Full list of author information is available at the end of the article
} 


\section{Background}

Biotechnology industry development over the last years made quality assurance more stringent for pharmacy production [1]. As a tool to resolve process data distortion and prevent operator from accidently making mistakes, bioengineering solutions help to automate tasks, which results in rise of cultivation process performance and quality. To strengthen product quality, to more efficiently acquire coefficient values, to improve safety and flexibility of adaptive feedback control, the soft/noninvasive sensors [2] become a rational choice for development of sustainable engineering solutions. Implementation of feedback control system requires a feedback signal from soft sensors or estimators that provide parameters [3], which are unavailable to be directly measured online [4]. The control algorithm and the feedback signal consider the product and the main characteristics of bioprocess parameters-the biomass concentration and the specific growth rate $[5,6]$.

This study delves into biomass estimator development based on stoichiometric parameters and Luedeking-Piret model. The cell's yields and stoichiometry both form a generic information, which is an acceptable candidate to be included in estimators when the microorganisms culture does not change from experiment to experiment. Depending on stoichiometry, the estimator of biomass concentration can be used to automatically inject the inoculant solution at a predefined level of the optical density in bioreactor medium. At this point, cumulative oxygen uptake rate signal from an off-gas analyzer is informative to determine the biomass concentration.

The biomass estimator described in this study includes optimization algorithm, which returns the stoichiometric parameters of the controlled culture. The algorithm refers to several optimization criteria and is based on a gray box model originating from Luedeking-Piret model. Then offline maximization of entropy leads to satisfactory parameters values for estimation procedure, which is then applied to Escherichia coli bacteria and S. cerevisiae yeast cultures. In other words, the stoichiometry optimization algorithm must be performed once for each strain to determine the necessary coefficients. These coefficients can be later used in the subsequent experiments to estimate biomass concentration online, unless the strain does not change. Such offline analysis can be considered as an estimator tuning algorithm for a specific microorganisms' culture.

The "Materials and methods" section describes the materials, strains and the bioreactor system operating conditions. The "Comparative analysis of biomass estimators" section reviews literature references of the off-gas analysis approaches and introduces the motivation for this study. The "General mathematical model of stoichiometric parameters estimation" section layouts the derivation of the bioengineering approach for both the offline (stoichiometry) analysis and the online (biomass concentration) analysis stages. It also resolves a general formulation of the oxygen consumption for biomass maintenance coefficient, which is relevant for both E. coli and S. cerevisiae cultures. The "Experimental validation" section provides experimental proof of the developed stoichiometry coefficients offline identification and the biomass concentration online estimation algorithms. The "Conclusions" section discusses the results and concludes the final statements of this study.

\section{Materials and methods \\ Cell strain's}

Four types of strain cultivation were analyzed in this work to verify biomass estimation. S. cerevisiae (no DY7221) strain was used as representative of yeasts cells. The recombinant strains E. coli BL21(DE3) pET9aIdeS, E. coli BL21 (DE3) pET21-IFN-alfa-5 (cloning of fused gene into bacterial systems with strong bacteriophage T7 promoter, pET21a + plasmid) [7] and E.coli BL21(DE3) pLysS [8] were used in bacterial cultivations.

\section{Medium and culture conditions}

In order to check biomass estimator's reliability and accuracy, data were collected from different cell strains which have been cultivated in multiple different $R \& D$ laboratories, including the laboratory of bioprocessing modeling and management in Kaunas University of Technology. Saccharomyces cerevisiae (no DY7221) strain was cultivated in the standard nutrient medium (YPD) $[9,10]$, which contained $1 \%$ yeast extract, $2 \%$ Bacto peptone, and $0.1 \%$ glucose. The feed solution contained $600 \mathrm{~g} / \mathrm{kg}$ glucose which increased the solution density to $1.21 \mathrm{~g} / \mathrm{l}$.

The medium temperature was maintained at $30{ }^{\circ} \mathrm{C}$ and it was monitored by using temperature sensor "Pt100", and $\mathrm{pH}$ was kept constant at 4.9 by addition of $\mathrm{NaOH}(\mathrm{aq})$ [11]. Dissolved oxygen tension DOT in the bioreactor was measured by oxygen electrode Mettler Toledo and controlled by shifting stirrer speed from 230 to $600 \mathrm{rpm}$. The DOT set point was chosen as $30 \%$ of air saturation. The air flow was kept around $41 / \mathrm{min}$ and measured by a mass air flow sensor. The off-gas from bioreactor was measured online by BlueSens gas analyzer (BCpreFerm, BlueSens, Herten, Germany), which has $\mathrm{O}_{2}, \mathrm{CO}_{2}$ and pressure sensors. The culture broth mass was measured online with balanced reactor vessel which contained load cell weight sensor. The initial substrate concentration in the bioreactor was equal to zero, $S=0 \mathrm{~g} / \mathrm{kg}$. Hence, after inoculation the substrate solution feeding was started. The cultivation process was performed in $5 \mathrm{l}$ bioreactor. 
The cell strain of E. coli BL21 (DE3) pET21-IFN-alfa-5 was cultivated in 71 bioreactor. Cultivation medium was based on minimal mineral medium, which was made of $46.55 \mathrm{~g}$ potassium dihydrogen phosphate, $14 \mathrm{~g}$ ammonium phosphate dibasic, $5.6 \mathrm{~g}$ citric acid monohydrate, $3 \mathrm{ml}$ of concentrate antifoam, $35 \mathrm{~g}$ magnesium sulphate heptahydrate, $105 \mathrm{~g} \mathrm{D}(+)$ glucose monohydrate. The initial volume of medium was $3.7 \mathrm{~kg}$. At the cultivation process the environment parameters were kept constant. The temperature setpoint was $37{ }^{\circ} \mathrm{C}$, DOT set at $20 \%$ of air saturation and $\mathrm{pH}$ kept at $\mathrm{pH} 6.8$ by addition of $\mathrm{NaOH}(\mathrm{aq})$. The stirrer rpm range was from 800 to $1200 \mathrm{rpm}$, the air flow rage was from 1.75 to $3.75 \mathrm{l} /$ min. In order to increase oxygen transfer rate during cultivation process, pure oxygen flow was provided to bioreactor at range from 0 to $7.5 \mathrm{l} / \mathrm{min}$. The off-gas from bioreactor was measured online by BlueSens.

The other cell strain of E. coli BL21 (DE3) pET9a-IdeS was cultivated in 151 bioreactor. Cultivation medium based as minimal mineral medium. At the cultivation process the environment parameters: temperature set point was $37^{\circ} \mathrm{C}$, DOT set at $30 \%$ of air saturation and $\mathrm{pH}$ kept at $\mathrm{pH} 6.98$ by addition of $\mathrm{NaOH}(\mathrm{aq})$. The stirrer $\mathrm{rpm}$ range was from 300 to $750 \mathrm{rpm}$, the air flow range was from 0.3 to $15 \mathrm{l} / \mathrm{min}$. During the cultivation process pure oxygen flow was provided to bioreactor at range from 0 to $7.5 \mathrm{l} / \mathrm{min}$. The off-gas from bioreactor was measured online by BlueSens.

For diversity of validation, the fourth cell strain was $E$. coli (BL21(DE3) pLysS) [8]. The cultivation medium used as minimal mineral medium composed with $\left(\mathrm{NH}_{4}\right)_{2} \mathrm{SO}_{4}$, $2.46 \mathrm{~g} / \mathrm{l} ; \mathrm{NH}_{4} \mathrm{Cl}, 0.5 \mathrm{~g} / \mathrm{l} ; \mathrm{NaH}_{2} \mathrm{PO}_{4} \times \mathrm{H} 2 \mathrm{O}, 3.6 \mathrm{~g} / \mathrm{l} ; \mathrm{Na}_{2} \mathrm{SO}_{4}$, $2 \mathrm{~g} / \mathrm{l} ; \mathrm{K}_{2} \mathrm{HPO}_{4}, 14.6 \mathrm{~g} / \mathrm{l} ;\left(\mathrm{NH}_{4}\right)_{2}$-citrate, $1 \mathrm{~g} / \mathrm{l} ; 1 \mathrm{M} \mathrm{MgSO}_{4}$ solution, $5 \mathrm{ml} / \mathrm{l}$; trace elements solution, $2 \mathrm{ml} / \mathrm{l}$; and no glucose. Initial masses of all cultures were $5 \mathrm{~kg}$. The glucose solution and initial substrate concentration at the bioreactor used same as at cultivation with yeasts, $\mathrm{pH}$ kept constant at $\mathrm{pH} 7$ and temperature was regulated to $30{ }^{\circ} \mathrm{C}$. Dissolved oxygen tension DOT was measured by an amperometric oxygen electrode (Mettler-Toledo) and the DOT set point was $30 \%$ of the saturation. The size of bioreactor was $15 \mathrm{l}$ working volume (Biostat C, Sartorius Stedim Biotech) and the stirrer speed varied from 100 to $1400 \mathrm{rpm}$.

\section{Comparative analysis of biomass estimators}

In order to adaptively control and monitor chemical or biotechnological process, it is mandatory to implement a data collection system that provides desired variables at real time with acceptable precision and performance. This requires corresponding equipment, which may be unaffordable, not implementable in system or the required instrument doesn't exist. Hence, the better alternative is to use soft or noninvasive sensors, which collect measurable variables and estimate unmeasurable parameters $[2,12]$. Especially in biotechnology processes, there are complex relationships between process and variables, so the best way to infer online unmeasurable parameters is to use corresponding estimators [4].

Over time, the studies of both bioprocesses and industrial production perspectives have shown that a biomass estimator requires data, which is closely related to biomass growth rate and biomass concentration. It can be indirectly measured online, with well-established and validated devices and soft sensors [4, 13], which are still in development. Oxygen uptake rate (OUR) and carbon dioxide production rate (CPR) are directly related to biomass growth rate and biomass concentration [14, 15]. Oxygen uptake rate (OUR) and carbon dioxide production rate $(\mathrm{CPR})$ data for estimator must be computed from online signals that are reliable and measured directly in bioreactor system. These signals are the concentration of $\mathrm{O}_{2}$ and $\mathrm{CO}_{2}$ in the off-gas [16]. The proposed noninvasive biomass concentration estimation procedure was intentionally made to not depend on the selection of bioprocess/bioreactor parameters. The approach is valid for aerobic cultures as long as it is possible to obtain the off-gas measurements of sufficient quality.

The main model, dedicated to biomass concentration estimation in this work, is a Luedeking-Piret model derived from the stoichiometric equations for oxygen consumption. It represents relationship between biomass $X$ growth/maintenance and oxygen uptake rate in bioreactor $[14,15]$ :

$$
\operatorname{OUR}(t)=\alpha \cdot X^{\prime}(t)+\beta \cdot X(t)
$$

Stoichiometric coefficients $\alpha$ and $\beta$ represent cell's metabolisms of oxygen consumption and correspond to the yield coefficients of these biochemical conversions. In Eq. (1) coefficient $\alpha$ means specific cell's oxygen consumption yield $\left(\alpha \equiv Y_{\mathrm{O}_{2} / \mathrm{X}}\right)$ for growth and $\beta$ is a model parameter termed as oxygen consumption for maintenance $\left(\beta \equiv \mathrm{m}_{\mathrm{o}_{2} / \mathrm{X}}\right)$ [17-20]. The generic structure of the Eq. (1) that describes the process does not include any strain specific information and there are no any initial conditions assumed for the values of both $\alpha$ and $\beta$.

Simutis and Lübbert (2006) improved a hybrid model estimator [21]. The main improvement of a dynamical mathematical model was a modification of mass balance equation to the new one, which was based on the oxygen uptake rate OUR, the carbon dioxide rate CPR and the base consumption rate BCR [22]. In order to further improve hybrid model's capacity, Kalman filter (EKF) was introduced to biomass estimations [23]. The new improved hybrid model produced better results and 
accuracy, but general drawbacks remained, estimator's complexity, a lot of data required for artificial neural network training and biomass estimation offline with a large execution duration [22-24]. In 2010, Simutis and Lübbert improved biomass estimator with cumulative variables that made model more conventional. The estimator procedure was transformed to a simpler system.

When comparing stoichiometry biomass estimators' mathematical models to the hybrid model estimator approaches, the latter contains more main state variables: biomass $(X)$, oxygen uptake rate (OUR), specific biomass growth rate $(\mu)$, broth weight $(\mathrm{w})$, carbon dioxide production rate (CPR), base consumption and other model coefficients. Additionally, additional equations and a fuzzy expert system are required. The latter gives an input to the combination of a dynamical mathematical model (DMM) represented by a set of nonlinear ordinary differential equations with an artificial neural network (ANN) [24]. The main advantage of the stoichiometry biomass estimator, compared to hybrid model, is its simplicity and accuracy. As hybrid model consists of several modeling systems, a common problem of estimation arrives from artificial neural network (ANN) training [21, 23, 24]. Meanwhile, stoichiometry biomass estimator was based only on OUR and stoichiometric parameters $\alpha$ and $\beta$, which both were kept static for a particular cell strain. This led to ability to calculate biomass online $[14,22-24$, 28]. A general comparison of different biomass estimators is presented in Fig. 1. This work's biomass estimation approach is depicted by Fig. 1d. The estimation methods, which are based on gas consumption stoichiometry, are shown in Fig. 1e, f. The main differences consist of the approach picked, its complexity and the number of input signals and prerequisite parameters or initial conditions required. The main purpose of this paper is to show that biomass estimation can be treated from the fundamental point of view based on the stoichiometry Eq. (1). The idea comes from entropic and Bayesian inference approaches involving integral optimizations [29, 30]. The focus lays on the implementation, which can be not only used in scientific R\&D laboratories, but also on the industrial plants level.

This paper presents a generic biomass estimation routine that is suitable for determination of biomass state in high diversity of bioreactors (Fig. 2) with potentially wide variety of industrial microorganisms. Prior to biomass determination, it is necessary to identify cell strain's stoichiometry parameters $\alpha$ and $\beta$, which both describe oxygen consumption by a microbial culture. This is accomplished by offline analysis Fig. 3 (stage A).

Afterwards, industrial scale cultivation processes reuse information about strain information for corresponding biomass concentration estimation in online analysis (stage B), as shown in Fig. 3. In order to achieve better accuracy at strain stoichiometry analysis during upstream development, it is recommended to identify $\alpha$ and $\beta$ parameters at the laboratory scale bioreactors, Fig. 3 (stage A). This way, strain stoichiometry analysis, based on "ground-truth" of stage A, is economically beneficial, and data from cultivation process consists of less disturbances in more flexible control environment.

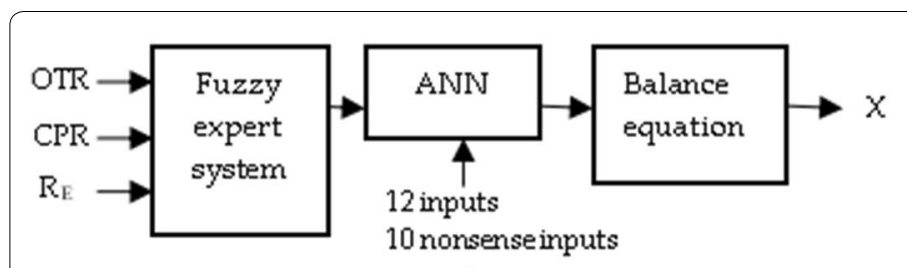

a
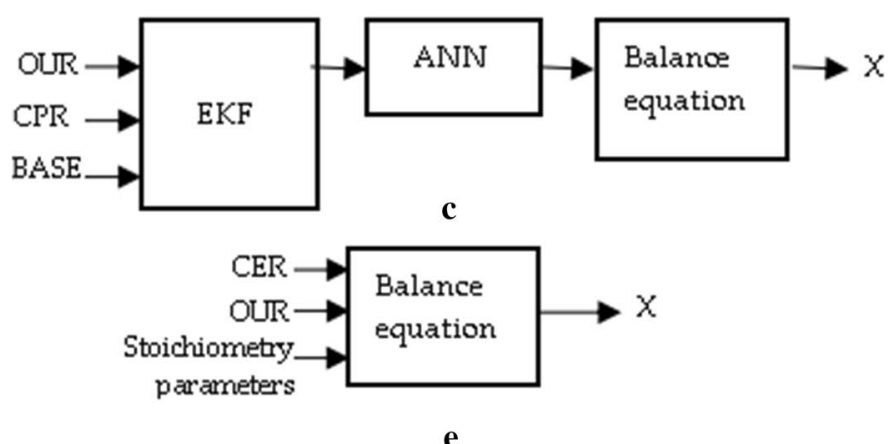

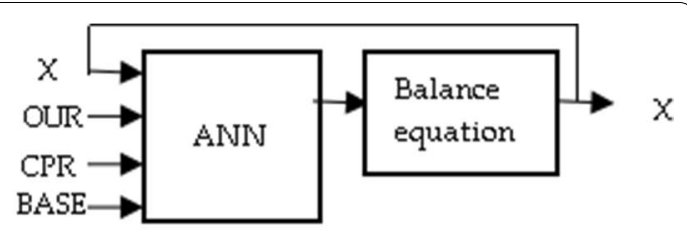

b

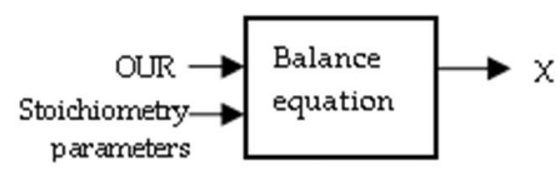

d

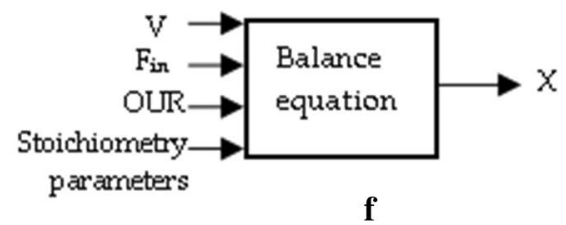

Fig. 1 Comparison of biomass estimators: a Lübbert [21], b Achle [25], c Simutis [22], d biomass estimation of this text, e Davis [26], f Barrigon [27] 


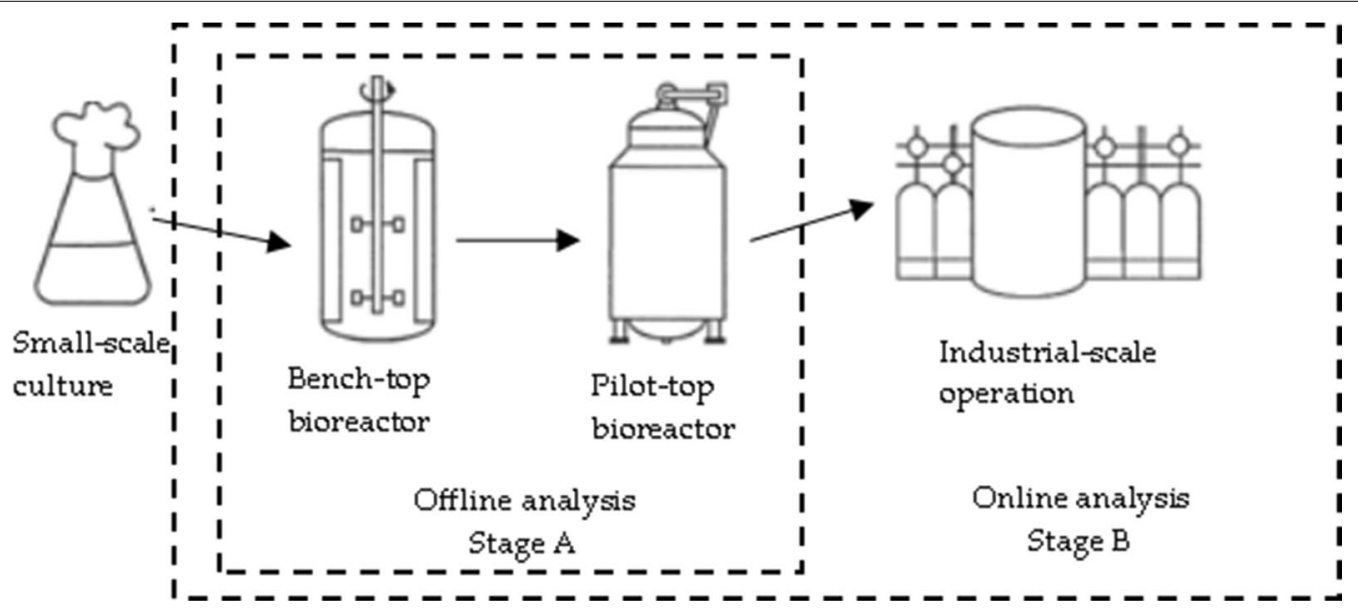

Fig. 2 Bioprocess technology development workflow

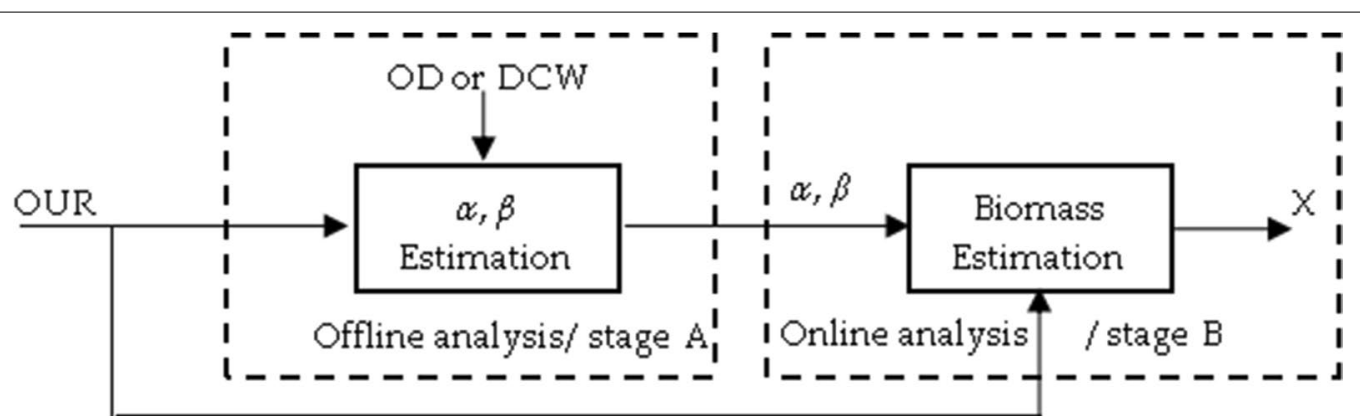

Fig. 3 Biomass estimator's structure scheme of different estimation stages

\section{General mathematical model of stoichiometric parameters estimation}

During the cultivation process, the real-time data collected from the devices has interference and disturbances, which may cause distortion of parameters and estimated values [14]. Simutis and Lübbert [4] stated "the reason for cumulating the original signals is to improve the signal-to-noise ratio (SNR) and thus increasing the information content about the process. Additionally, as the biomass and its metabolic products are accumulated during the cultivation, these masses are better correlated with the cumulative signals of OUR and CPR". The main method of the current text is also based on the integral approach, which can be considered as a filter eliminating noise [22]. Hence, the Luedeking-Piret model Eq. (1) outcomes are being protected from disturbances by integrating it:

$$
\int_{t_{0}}^{t} \operatorname{OUR}\left(t^{*}\right) \mathrm{d} t^{*}=\alpha \cdot \int_{t_{0}}^{t} X^{\prime}\left(t^{*}\right) \mathrm{d} t^{*}+\beta \cdot \int_{t_{0}}^{t} X\left(t^{*}\right) \mathrm{d} t^{*} .
$$

According to data from bioprocesses and previous experience, the stoichiometric parameter $\beta$ is assumedly not a process constant. During the cultivation, parameter $\beta$-oxygen maintenance coefficient for biomass, increases due to biomass concentration growth. The phenomenon of increasing value of parameter $\beta$ can be explained by the fact that the consumption of oxygen for biomass maintenance also includes the generation of the product and other factors. Such situation occurs at the end of the exponential phase of a microbial cultivation (for recombinant protein synthesis) when the induction (e.g., with isopropyl-D-1-thiogalactopyranoside/IPTG) is performed and the synthesis of the product increases noticeably. As a result, oxygen consumption for biomass maintenance also increases $[31,32]$. The parameter $\beta$ consists of two additive terms

$$
\beta=\frac{1}{Y_{X O}}+\frac{1}{Y_{P O}}
$$

where $Y_{X O}$ is oxygen consumption for cells breathing and $Y_{P O}$ is oxygen consumption for product formation. 
Consequently, biomass has linear/polynomial relationship to parameter $\beta$ which is directly dependent on biomass concentration.

The observational data used for proposed biomass estimation was obtained from the processes that involve recombinant protein expression. As it can be seen from the Eq. (3), the parameter $\beta$ accounts for both, biomass and product, yields. This parameter may exhibit different behavior depending on the process phase and the strain/ product involved. However, comprehensive comparison of various strains with respect to the impact, that particular product has on the biomass estimator performance, or to explore the effect on metabolic noise debugging in strain engineering, goes beyond the scope of this study.

To remove the assumption that the stoichiometric parameter $\beta$ is a function of a biomass, this parameter is expressed as a function of time in the mathematical model. Hence, Eq. (3) is rewritten to linear regression of time:

$$
\beta=k_{1} * t+k_{2}
$$

where $k_{1}$ and $k_{2}$ are linearly dependent mathematical coefficients. When bioprocess is at lag phase or early phase of exponential growth (when biomass concentration is relatively low), the $\beta$ parameter is extremely small and negligible. Only after induction or specific value of biomass concentration, oxygen consumption for maintenance becomes appreciable. Hence, during a time prior to fact when the Eq. (4) comes into effect, the parameter $\beta$ should be set to zero in the estimation procedure. At that moment the biomass concentration reaches a value from which the consumption of oxygen for biomass maintenance becomes significant:

$$
\begin{aligned}
& 0=k_{1} * t_{i}+k_{2} \stackrel{\text { yields }}{\longrightarrow} \\
& k_{2}=-k_{1} * \mathrm{t}_{\mathrm{i}} .
\end{aligned}
$$

Then parameter $\beta$ becomes

$$
\beta=k_{1} * t-k_{1} * t_{i}
$$

where $t_{i}$ is the duration from cultivation process start to the time when amount of biomass reaches value resulting in appreciable oxygen maintenance, or when induction is performed and product formation noticeably increases, or when stoichiometry parameter $\beta$ is no longer zero [9, $31,32]$. In order to have full mathematical model formula, main balance Eq. (2) has parameter $\beta$ replaced in the linear regression Eq. (7):

$\int_{t_{0}}^{t} \operatorname{OUR}\left(t^{*}\right) \mathrm{d} t^{*}=\alpha \cdot \int_{t_{0}}^{t} X^{\prime}\left(t^{*}\right) \mathrm{d} t^{*}+\int_{t_{0}}^{t} k_{1} \cdot\left(t^{*}-t_{i}\right) \cdot X\left(t^{*}\right) \mathrm{d} t^{*}$.

\section{Offline analysis of stoichiometry parameters (stage A)}

Prior to the estimation of the biomass, specific cell strain's stoichiometric parameters must be identified during offline analysis. There are few compulsory inputs to approach this task.

- Model fitting procedure requires offline observations: dry cell weight (DCW) or optical density OD value (in o.u.) multiplied by a coefficient of biomass concentration (approximately $0.4 \mathrm{~g} / \mathrm{l} / \mathrm{o}$.u.) [33];

- Process duration time since cells' inoculation to bioreactor, in hours;

- Oxygen uptake rate (OUR) data since the inoculation;

For model fitting a chosen mathematical expression is equated to gray box model since the collected experimental data is combined with fundamental knowledge about bioprocess [34]. Considering that the bioprocess consists of two main parts, prior to induction and after it, the parameters fitting procedure is based on two independent gray box models. The first one covers the first two cultivation process phases: the lag and exponential. During these phases the amount of biomass is low and materials, resources concentrate to biomass growth [35]. Hence, oxygen requirement for biomass maintenance is minimum and stoichiometric parameter $\beta$ is negligible:

$$
\int_{t_{0}}^{t_{i}} \operatorname{OUR}\left(t^{*}\right) \mathrm{d} t^{*}=\alpha \cdot \int_{t_{0}}^{t_{i}} X^{\prime}\left(t^{*}\right) \mathrm{d} t^{*}
$$

In the Eq. (9) the variable $t_{i}$ is the time of the induction or the time when biomass reaches a quantity where oxygen usage for maintenance is appreciable. The second cultivation stage represents the biomass growth deceleration and increasing product formation. In this cultivation phase, additional term comes into effect, oxygen consumption for maintenance and product formation, known as stoichiometric parameter $\beta$. To properly describe second gray box model, the induction time or time when biomass concentration reaches specific amount must be identified. Throughout this period the maintenance term becomes significant and can't be negligible. After applying maintenance parameter to a model, the second gray box model's expression is generalized to

$\int_{t_{0}}^{t} \operatorname{OUR}\left(t^{*}\right) \mathrm{d} t^{*}=\alpha \cdot \int_{t_{0}}^{t} X^{\prime}\left(t^{*}\right) \mathrm{d} t^{*}+\int_{t_{0}}^{t} k_{1} \cdot\left(t^{*}-t_{i}\right) \cdot X\left(t^{*}\right) \mathrm{d} t^{*}$.

In summary, the Eqs. (10) and (11) both yield the conditional definition of cumulative oxygen uptake rate function: 


$$
\left\{\begin{array}{l}
\operatorname{cOUR}\left(t \leq t_{i}\right) \equiv \int_{t_{0}}^{t} \operatorname{OUR}\left(t^{*}\right) \mathrm{d} t^{*}=\alpha \cdot\left(X(t)-X_{0}\right) ; \quad t \leq t_{i} ; \\
\operatorname{cOUR}\left(t>t_{i}\right) \equiv \int_{t_{0}}^{t} \operatorname{OUR}\left(t^{*}\right) \mathrm{d} t^{*} \approx \alpha \cdot\left(X(t)-X_{0}\right)+\sum_{l=i}^{m} k_{1} \cdot\left(t_{l}-t_{i}\right) \cdot X\left(t_{l}\right) \cdot \Delta t_{l, l-1} ; \quad t>t_{i} .
\end{array}\right.
$$

In Eq. (11) the last sum of products is the expression of left Riemann sum [36], i.e. $\int_{t_{0}}^{t} k_{1} \cdot\left(t^{*}-t_{i}\right) \cdot X\left(t^{*}\right) \mathrm{d} t^{*} \approx$ $\sum_{l=i}^{m} k_{1} \cdot\left(t_{l}-t_{i}\right) \cdot X\left(t_{l}\right) \cdot \Delta t_{l, l-1}$, when time's $t$ sample is indexed by $m$. Discrete DCW values define variable $X_{l} \equiv X\left(t_{l}\right)$, where $l \in\left[1, n_{m}\right], n_{m}$ is the total number (e.g. hourly) of offline sampling intervals with index $m$ and $X_{0} \equiv X\left(t_{0}\right)$ is an initial biomass concentration after inoculation into bioreactor.

\section{Procedure for offline analysis of stoichiometry parameters}

The prediction value of the cumulative OUR model [37] for Eq. (11) is

$$
\operatorname{cOUR}_{m} \equiv\left\{\begin{array}{l}
\operatorname{cOUR}\left(t_{m} \leq t_{i}\right)=\alpha \cdot\left(X_{m}-X_{0}\right) \\
\operatorname{cOUR}\left(t_{m}>t_{i}\right)=\alpha \cdot\left(X_{m}-X_{0}\right)+\sum_{l=i}^{m} k_{1} \cdot\left(t_{l}-t_{i}\right) \cdot X_{l} \cdot \Delta t_{l, l-1} ;
\end{array}\right.
$$

Then the posterior distribution for $m$-th offline sample is

$$
P_{\text {posterior }}\left(\mathrm{COUR}_{m}\right) \sim N\left(\mathrm{COUR}_{m}, \sigma_{c \mathrm{OUR}}^{2}\right),
$$

where every sampled prediction $m$ has constant variance $\sigma_{c O U R}^{2}$.

Prior distribution also has the form of Gaussian distribution [38]

$$
P_{\text {likelihood }}\left(c O U R_{m}\right) \sim N\left(c O U R_{m}^{*}, \sigma_{c O U R, m}^{2}\right),
$$

where $c O U R_{m}^{*}$ is the $m$-th observation value of the cumulative OUR and its unique variance is $\sigma_{c O U R, m}^{2}$.

In previous work [37] the uncertainty of prior distribution was assumed to be equal to the square of observed value, i.e. $\sigma_{c O U R, m}^{2}$ was assumed to be proportional to $c O U R_{m}^{* 2}$. However, this assumption is not quite rational from practical considerations based on this work experience when deriving a generic estimator for both $E$. coli and yeast cultures. It appears that the assumption of $\sigma_{c O U R, m}^{2} \sim \operatorname{cOU} R_{m}^{* 2}$ is just a special case, which has even more general form. Interestingly this form matches the form of Monod formulation [39] applied to uncertainty, i.e.

$$
\sigma_{c O U R, m}^{2}=\sigma_{\max }^{2} \frac{X_{m}^{2}}{K_{X^{2}}+X_{m}^{2}},
$$

where scenario with $K_{X^{2}}=0$ resembles least squares approach, i.e. all samples' relative weights become equal, and $K_{X^{2}} \rightarrow \infty$ means that $\sigma_{c O U R, m}^{2} \sim \mathrm{COUR}_{m}^{* 2}$ as in previous work [37]. In other words, empirical coefficient $K_{X^{2}}$ is a "weight" coefficient between the two additive terms of optimization criterion. The first term is the least squares criterion and the other is "squared MAPE" criterion as in [37]. Another note about Monod Eqs. (15) and (12) is that the relationship of $\sigma_{c O U R, m}^{2} \sim \sigma_{X, m}^{2}$ is valid, i.e. the uncertainty of cumulative OUR is proportional to the uncertainty of biomass variable.

To rationally prepare Eq. (15) for simplified numeric operations avoiding infinities when estimating values, an intrinsic variable $K_{\text {exp }}$ expression replaces $K_{X^{2}} \rightarrow \frac{1-K_{\exp }}{K_{\exp }}$ and transforms Eq. (15) to

$$
\sigma_{c O U R, m}^{2}=\sigma_{\max }^{2} \frac{X_{m}^{2}}{\frac{1-K_{e x p}}{K_{\text {exp }}}+X_{m}^{2}} \stackrel{\text { yields }}{\longrightarrow} \sigma_{\max }^{2} \frac{X_{m}^{2} \cdot K_{\exp }}{1-K_{\exp }+X_{m}^{2} \cdot K_{\exp }},
$$

The fact, that $\sigma_{\max }^{2}$ and $K_{\exp }$ both are positive scalar values and do not depend on the index $m$ of a sampling interval, allows to simplify Eq. (16) to

$$
\sigma_{\text {cOUR }, m}^{2} \sim \frac{X_{m}^{2}}{1-K_{\exp }+X_{m}^{2} \cdot K_{\exp }} .
$$

Equation (17) exposes the physical meaning of $K_{\text {exp }}$. The scenario with $K_{\text {exp }}=0$ recovers $\sigma_{c O U R, m}^{2} \sim X_{m}^{2}$ as in [37]. The scenario with $K_{\exp }=1$ recreates the least squares method as in $[38,40]$. Both scenarios show that $K_{\text {exp }}$ is an exponential weight, which constructs a hybrid criterion for both least squares and the MAPE squared. Later in the text, the experimental validation will show that there exists a rational empirical value of $K_{\text {exp }}$, which enables estimation of the biomass concentration, with an acceptable precision, for both yeast and $E$. coli cultures since the beginning of the cultivation right after the culture was inoculated to a bioreactor.

After gray box model is identified and hybrid criterion derived, the next step is to use optimization approach to find the stoichiometry parameters. The main equation solving for unknown parameters comes from the maximization of entropy [37, 39] based on Eqs. (13), (14) and (17) 


$$
\begin{aligned}
S= & -\sum_{m=1}^{m \leq i} \frac{\left(c O U R_{m}-\alpha \cdot\left(X_{m}-X_{0}\right)\right)^{2}}{\frac{X_{m}^{2}}{K_{\text {exp }} \cdot X_{m}^{2}+\left(1-K_{\text {exp }}\right)}} \\
& -\sum_{m=i+1}^{n_{m}} \frac{\left(c O U R_{m}-\alpha \cdot\left(X_{m}-X_{0}\right)-\sum_{l=1}^{m} k_{1} \cdot\left(t_{l}-t_{i}\right) \cdot X\left(t_{l}\right) \cdot \Delta t_{l, l-1}\right)^{2}}{\frac{X_{m}^{2}}{K_{\text {exp }} \cdot X_{m}^{2}+\left(1-K_{\text {exp }}\right)}} .
\end{aligned}
$$

Hence, at the optimization method, which is shown at the Eq. (18), the whole $S$ expression is maximized, and unknown stoichiometry parameters are found by solving partial derivative of Eq. (18) with respect to $\alpha$ and $k_{1}$

$$
\left\{\begin{array}{l}
\frac{\partial S}{\partial \alpha}=0 \\
\frac{\partial S}{\partial k_{1}}=0 .
\end{array}\right.
$$

Equation (19) yields the linear system of two equations

$$
\left\{\begin{array}{l}
\alpha \cdot B+k_{1} \cdot C=A ; \\
\alpha \cdot E+k_{1} \cdot F=D ;
\end{array} .\right.
$$

where Eq. (20) parameters are:

$$
\begin{aligned}
& A=\sum_{m=1}^{n_{m}} \frac{\left(X_{m}-\mathrm{X}_{0}\right) \cdot c \mathrm{OUR} R_{m}}{\frac{X_{m}^{2}}{K_{\text {exp }} \cdot X_{m}^{2}+\left(1-K_{\text {exp }}\right)}} \\
& B=\sum_{m=1}^{n_{m}} \frac{\left(X_{m}-X_{0}\right)^{2}}{K_{\text {exp }} \cdot X_{m}^{2}+\left(1-K_{\exp }\right)} ; \\
& C=\sum_{m=i+1}^{n_{m}} \frac{\left(X_{m}-X_{0}\right) \cdot \sum_{l=1}^{m}\left(t_{l}-t_{i}\right) \cdot X\left(t_{l}\right) \cdot \Delta t_{l, l-1}}{\frac{X_{m}^{2}}{K_{\text {exp }} \cdot X_{m}^{2}+\left(1-K_{\text {exp }}\right)}} ; \\
& D=\sum_{m=i+1}^{n_{m}} \frac{c O U R_{m} \cdot \sum_{l=1}^{m}\left(t_{l}-t_{i}\right) \cdot X_{l} \cdot \Delta t_{l, l-1}}{\frac{X_{m}^{2}}{K_{e x p} \cdot X_{m}^{2}+\left(1-K_{\text {exp }}\right)}} ; \\
& E=\sum_{m=i+1}^{n_{m}} \frac{\left(X_{m}-X_{0}\right) \cdot \sum_{l=1}^{m}\left(t_{l}-t_{i}\right) \cdot X_{l} \cdot \Delta t_{l, l-1}}{\frac{X_{m}^{2}}{K_{\text {exp }} \cdot X_{m}^{2}+\left(1-K_{\text {exp }}\right)}} ; \\
& F=\sum_{m=i+1}^{n_{m}} \frac{\left(\sum_{l=1}^{m}\left(t_{l}-t_{i}\right) \cdot X_{l} \cdot \Delta t_{l, l-1}\right)^{2}}{\frac{X_{m}^{2}}{K_{e x p} \cdot X_{m}^{2}+\left(1-K_{e x p}\right)}} .
\end{aligned}
$$

Equations (20)-(26) finalizes the offline estimation of stoichiometry parameters, which are then later used for online estimation of biomass concentration. However, the variable $t_{i}$ has no direct meaning with yeast cultures, so it must be dealt with separately. First, the specific time when the maintenance coefficient becomes appreciable is analyzed in the next subsection.

\section{Identification of yeasts' specific time for maintenance}

Variable $t_{i}$ at Eq. (12) is the time of induction or the time when biomass concentration reaches a specific amount when oxygen maintenance for cells becomes non negligible. In the case of cultivation processes of E. coli, the induction time is known, i.e. it can be defined by the time moment when IPTG solution is injected into bioreactor. In the cultivation process of $S$. cerevisiae yeasts the IPTG solution was not used. Hence, the variable $t_{i}$ defines the time when biomass concentration reaches a specific value when maintenance coefficient becomes noticeable. The search for $t_{i}$ utilizes the convex optimization method and maximization of entropy [37, 41]. The optimization procedure is depicted in Fig. 4.

The knowledge of the specific time $t_{i}$ enables the biomass concentration estimation. However, the specific time $t_{i}$ is not known in advance prior to online experiment with yeast cells, because it has just a theoretical meaning in this case. Therefore, a generic relationship between the maintenance coefficient value and the biomass concentration will be inferred in the next subsection. Such a generic form of maintenance coefficient will enforce online estimation without dependence on the type of the microbial culture. Moreover, the value of the specific time $t_{i}$ becomes irrelevant for the online estimation procedure.

\section{Identification of maintenance coefficient parts}

After optimization of stoichiometry parameters, which had determined unknown parameters of the mathematical method, the next step is to validate those identified parameters with experimental data. Prior to comparison of theoretical and experimental data, the mathematical model, as in Eq. (7), must be reconstructed so that $\beta$ is no longer a function of time and still satisfies the actual behavior of biotechnological process. The stoichiometric parameter $\beta$ directly depends on biomass concentration

$$
\beta(X) \equiv \beta(X(t))=k_{\beta 2} \cdot X^{2}(t)+k_{\beta 1} \cdot X(t)+k_{\beta 0} ;
$$




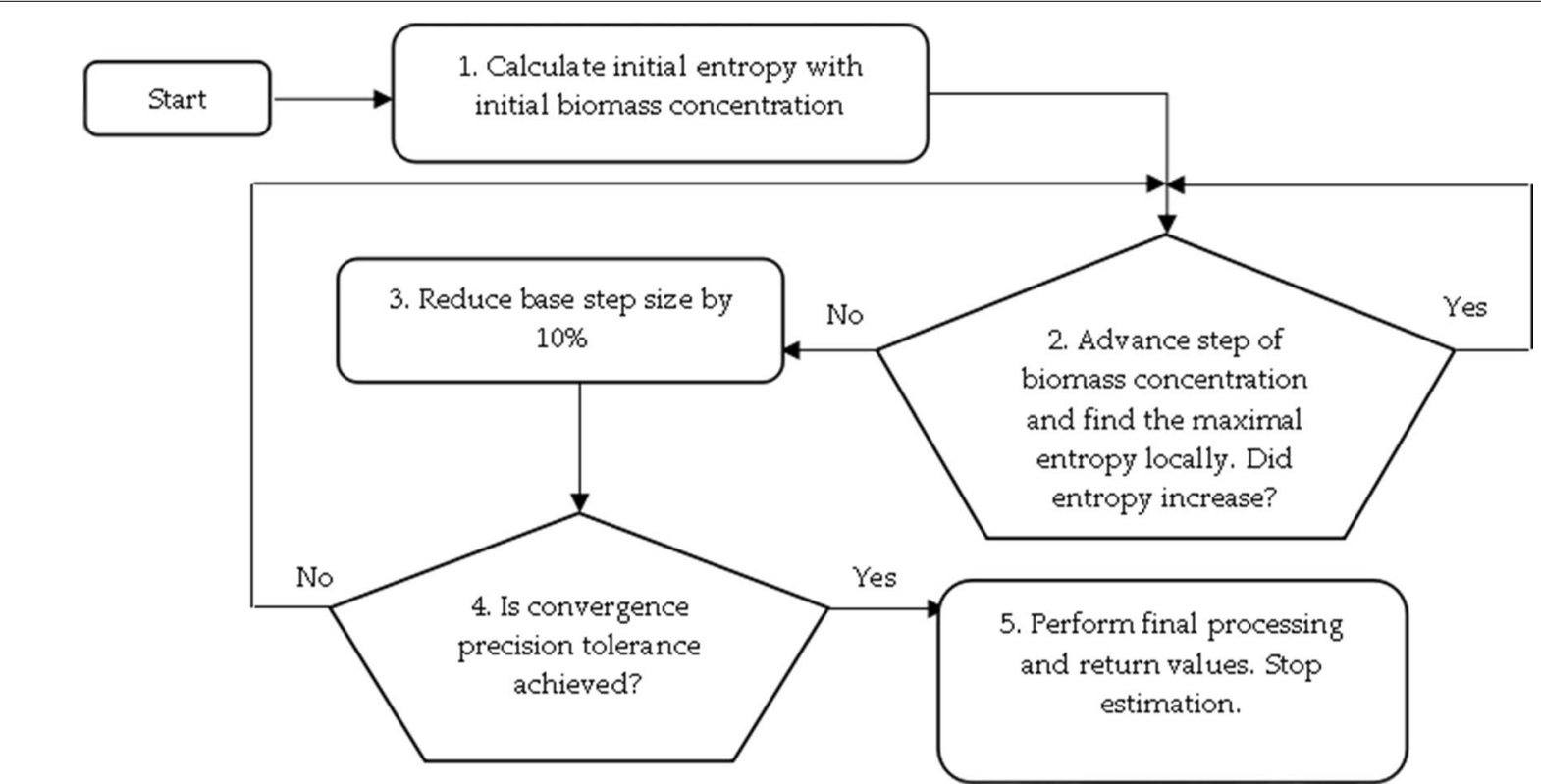

Fig. 4 The workflow of structural scheme for parameter identification
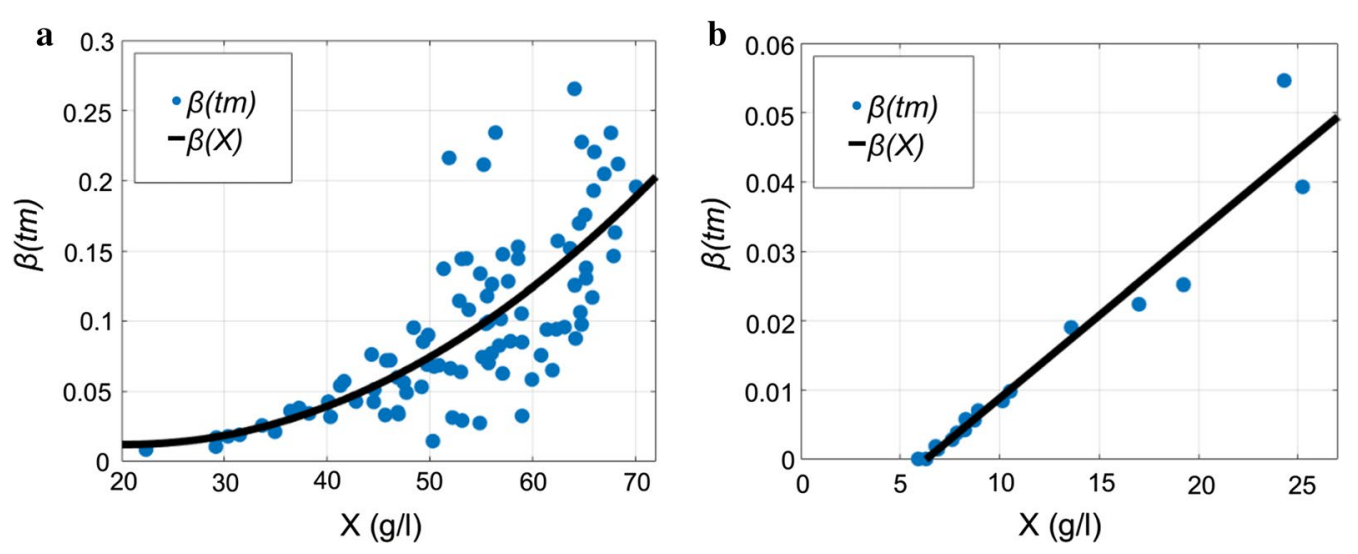

Fig. 5 Dependence of oxygen consumption for maintenance on biomass concentration, a E. coli, b Saccharomyces cerevisiae

The expression of parameter $\beta(X)$ represents a parabola regression of biomass in the case of the E. coli strain Fig. 5a. Meanwhile, S. cerevisiae oxygen consumption for maintenance is dependent linearly on biomass concentration, thus $k_{\beta s 2}=0$,

$$
\beta_{\text {Saccharomyces }}(X) \equiv \beta_{\text {Saccharomyces }}(X(t))=k_{\beta s 1} \cdot X(t)+k_{\beta s 0} ;
$$

In Eqs. (27) and (28) regression coefficients connect maintenance coefficient $\beta$ to biomass variable. In both culture cases, stage A helps to obtain $\beta$ values from linear regression based on Eq. (7) output

$$
\beta\left(X_{m}\right) \cong \beta\left(t_{m}\right)=k_{1} *\left(t_{m}-t_{i}\right) .
$$

The assumed relationship of $\beta(X)$ considering biomass concentration is presented in Fig. 5.

According to data from cultivation processes of E. coli in Fig. 5, the stoichiometric parameter of cell maintenance can be assumed as directly dependent on biomass in parabolic manner. At the cultivation processes of $E$. coli, the induction of IPTG, which initiates product synthesis, may cause nonlinear dependence of oxygen consumption on biomass maintenance. Based on Eqs. (27) and (28), it is possible to calculate strain's specific biomass concentration $\left(X_{\text {specific }}\right)$ when oxygen consumption for maintenance is no longer negligible. This is done by setting Eqs. (27) and (28) to zero and solving them for the specific biomass concentration $X_{\text {specific }}$ 


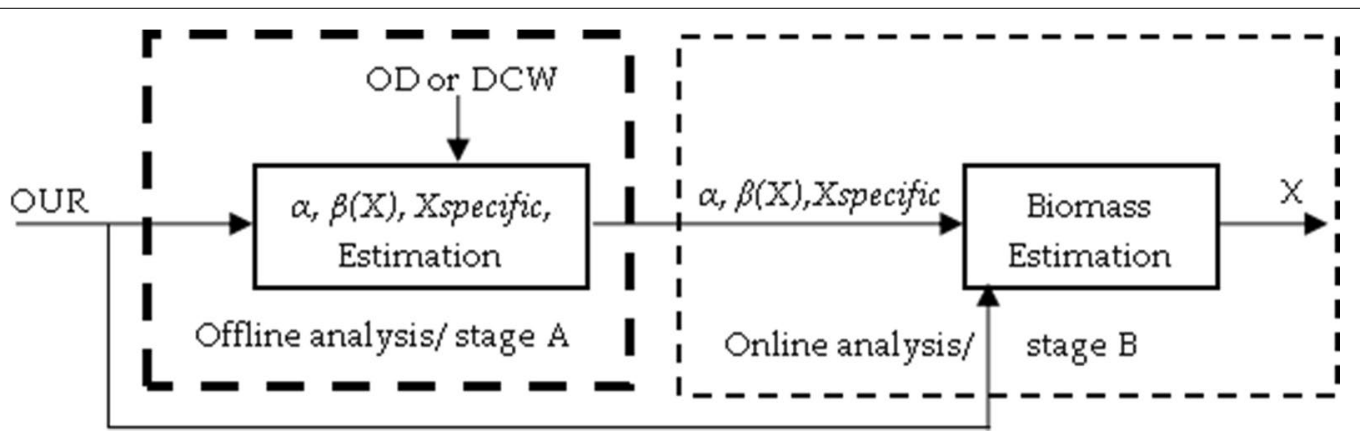

Fig. 6 Biomass estimation workflow

$$
\beta\left(X_{\text {specific }}\right) \equiv \beta(X(t))=0 ;
$$

The workflow of both stoichiometry and biomass estimations improves structure, as in Fig. 3, to the shape of the one in Fig. 6.

The solution of Eq. (30) identifies the specific biomass concentration $X_{\text {specific }}$ and finalizes the offline estimation of stoichiometry coefficients for a strain. After the stoichiometry coefficients are found in stage A, a generic procedure for online biomass estimation can be performed independently on the knowledge of bioreactor parameters. In conclusion, $\beta$, as in Eq. (27), transforms Eq. (1) into
Prior to the specific biomass $X_{\text {specific }}$ level is reached, i.e. when oxygen consumption for maintenance is very low or negligible, biomass state estimator equation is

$$
X_{m}=\frac{c O U R_{m}}{\alpha}+X_{0} .
$$

After biomass concentration exceeds $X_{\text {specific }}$ during the second scenario, i.e. oxygen consumption becomes noticeable, the stoichiometric parameter $\beta$ comes into effect as a function of biomass concentration. Equation (12) helps to derive the approximate estimator for biomass state, as follows

$$
\left\{\begin{array}{l}
\operatorname{OUR}(t)=\alpha \cdot X^{\prime}(t)+k_{\beta 2} \cdot X^{3}(t)+k_{\beta 1} \cdot X^{2}(t)+k_{\beta 0} \cdot X(t), X(t)>X_{\text {specific }} \\
\operatorname{OUR}(t)=\alpha \cdot X^{\prime}(t), X(t) \leq X_{\text {specific }}
\end{array}\right.
$$

In spite of the fact that Eq. (31) form is the third order function, it is still the same equation as Eq. (1). However, it was inferred by the estimation procedure and the observation data in Fig. 5. Variable $\beta$ manipulation compensates the effect of biomass concentration $X$ on $\beta$ and makes all Eq. (31) coefficients linearly dependent and constant throughout the course of the experiment. Eventually, this serves as a prerequisite to the simplified generic procedure for estimation of biomass concentration, coming in the next subsection.

\section{Online estimation of biomass concentration (stage B)}

In this paper, estimation of biomass concentration is based on stoichiometric parameters and cumulative oxygen uptake rate $c O U R$. When stoichiometric parameters are discovered in stoichiometry estimation, stage $\mathrm{A}$, or it was given, only one input from bioreactor system, cumulative oxygen uptake rate, is necessary to estimate the biomass state. This procedure is depicted by stage B (online analysis) in Fig. 6. The block of "biomass estimation", Fig. 6, consists of two main scenarios which both return biomass concentration at a time instance with index $m$.

$$
X_{m} \cong \frac{c O U R_{m}-\sum_{l=i}^{m-1} \beta\left(X_{l}\right) \cdot X_{l} \cdot \Delta t_{l, l-1}}{\alpha}+X_{0}
$$

The variable $X_{0}$, as in Eqs. (32) and (33), is an initial biomass concentration at the time of inoculation into bioreactor. Its value can be either a dry biomass measurement value or optical density OD value (in o.u.) multiplied by a coefficient of biomass concentration (approximately $0.4 \mathrm{~g} / \mathrm{l} / \mathrm{o} . \mathrm{u}$.).

This subsection initializes the online biomass estimation procedure (Fig. 7), which can be used in biotechnological industrial practices. The suggested approach does not require the bioreactor-dependent parameters, it serves as a good candidate to be applied to more microbial strains and the experimental validation, in the coming section, will show that such an approach can be used for biomass estimation since the time moment of inoculation into bioreactor.

\section{Experimental validation}

Validation performance indicators

Both mean absolute error (MAE) and mean absolute percentage error (MAPE) were used as indicators to evaluate 


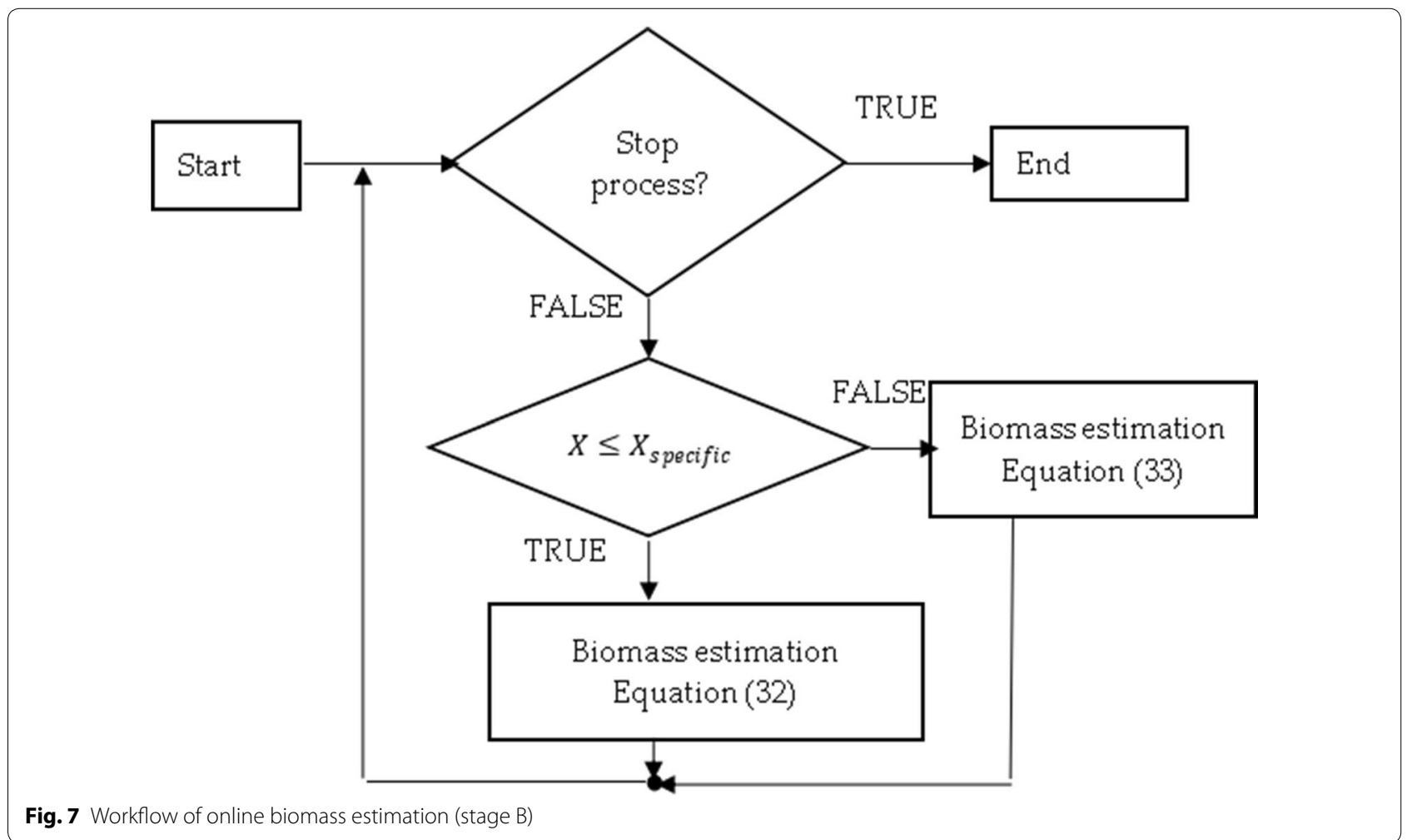

the estimation results. MAE and MAPE methods both evaluate the errors between estimated and observed biomass values of a cultivation process. MAE approach is defined as follows [42]:

$$
M A E=\frac{\sum_{i=1}^{n}\left|\hat{y}_{i}-y_{i}\right|}{n},
$$

where $n$ is the number of data counts, $\hat{y}_{i}$ is estimation result, which is compared to $y_{i}$, the observed value from the cultivation process. Mean absolute error represents average vertical distance between both values. MAPE method can be expressed as follows [43]:

$$
M A P E=\frac{100 \%}{n} \sum_{i=1}^{n}\left|\frac{\hat{y}_{i}-y_{i}}{y_{i}}\right| .
$$

The mean absolute percentage error is a statistical measure representing the accuracy of a forecast system, in percentage. Root mean square error represents the square root of residuals of the differences between predicted values and observed values. RMSE method's formula are as follows [42]:

$$
R M S E=\sqrt{\frac{\sum_{i=1}^{n}\left(\hat{y}_{i}-y_{i}\right)^{2}}{n}} .
$$

Table 1 Stoichiometric parameters of cell strains

\begin{tabular}{ll}
\hline Escherichia coli & Saccharomyces cerevisiae \\
\hline$a=1.01$ & $a=1.35$ \\
Confidence Interval $\mp 0.0186$ & Confidence Interval $\mp 0.149$ \\
$k_{\beta e 2}=7.2 \cdot 10^{-5}$ & $k_{\beta s 2}=0$ \\
$k_{\beta e 1}=-2.9625 \cdot 10^{-3}$ & $k_{\beta s 1}=2.3851 \cdot 10^{-3}$ \\
$k_{\beta e 0}=4.27047 \mathrm{~d} \cdot 10^{-2}$ & $k_{\beta \text { s0 }}=-1.5014 \cdot 10^{-2}$ \\
$X_{\text {specific }}=20.6 \mathrm{~g} / \mathrm{l}$ & $X_{\text {specific }}=6.29 \mathrm{~g} / \mathrm{l}$ \\
$K_{\text {exp }}=0.4$ & \\
\hline
\end{tabular}

\section{Comparative analysis of experimental results}

Experimental biomass measurements and data of cumulative oxygen uptake rate $c O U R$ from fed-batch experiments of E. coli and S. cerevisiae were taken from [8], experiments led by authors of this text and industrial R\&D laboratories. There were three cultivations of E. coli cells in 151 bioreactor with limited substrate feed [8] and two R\&D laboratory cultivations of $S$. cerevisiae yeasts in 51 bioreactor with limited substrate feed. Additionally, there was one cultivation of E. coli in 121 bioreactor with limited substrate feed and there were 15 cultivations in $5 \mathrm{l}$ bioreactor, out of which 7 cultivations were with dosed substrate feeding. As the first step, all cultivation data was analyzed in the stoichiometric parameters' 
estimation (stage A). The estimation procedure ignored both metabolism pathways, occurring during dosed substrate feed cultivations, and increasing product formation due to IPTG injections. The results of offline analysis of stoichiometric parameters are present in Table 1.

The tuning coefficient $K_{\exp }$ was identified empirically and its value of 0.4 showed acceptable outcome for the performed experiments. However, S. cerevisiae stoichiometric results come from just two cultivation experiments. Therefore, the results might still be improved when more experimental data becomes available in the future.

In industrial processes, strain's stoichiometric parameters are given, unless they were estimated using offline analysis, stage A. Then biomass concentration is calculated iteratively using both Eqs. (32) and (33) from cOUR signal (online analysis, stage B). This work's biomass estimation method used different cultivation experiments, with different cell strains, bioreactor volumes, type of substrates feeding solution, different IPTG induction time moment and their corresponding OD levels at IPTG injection, different substrate feeding limitations and different time of starting the substrate feed. Estimation results are shown in Table 2.
Seven experiments (\#5-\#11) were performed with dosed substrate feeding. Meanwhile the rest of experiments had limited feeding with various combinations of control strategies described in [37]: multiple different substrate limited feedings prior to induction and after it.

The overall average MAE of biomass estimation since inoculation is $1.1 \mathrm{~g} / \mathrm{l}$ and overall average MAE of biomass estimation since feed start is $1.41 \mathrm{~g} / \mathrm{l}$. The overall average MAPE of biomass estimation since inoculation is $7.28 \%$ and overall average MAPE of biomass estimation since feed start is $6.29 \%$. Overall average RMSE value of $S$. cerevisiae cultivations is $0.5 \mathrm{~g} / \mathrm{l}$. RMSE value of $E$. coli cultivations with limited substrate feeding is $1.26 \mathrm{~g} / \mathrm{l}$ and for cultivations with dosed substrate feeding is $2.44 \mathrm{~g} / \mathrm{l}$. RMSE value of $E$. coli cultivations before stationary phase, when DCW reaches $\sim 40 \mathrm{~g} /$ l (to compare with results in [22]) with limited substrate feeding, is $1.07 \mathrm{~g} / \mathrm{l}$ and for cultivations with dosed substrate feeding is $1.2 \mathrm{~g} / \mathrm{l}$. These results show that this approach improves the precision achieved in [22] without compromising the simplicity of the implementation. Offline analysis (stage A) execution lasted 2-15 $\mathrm{ms}$ and online analysis (stage B) calculations took $13-30 \mathrm{~ms}$ on a single core CPU in bioprocess engineering software tool dedicated for the purposes of this work. No initial conditions for numeric

Table 2 Analysis of experiments for biomass estimation

\begin{tabular}{|c|c|c|c|c|c|c|}
\hline No. & Strain & $\begin{array}{l}\text { Bioreactor } \\
\text { volume, I }\end{array}$ & $\begin{array}{l}\text { MAE since inoculation } \\
\mathrm{g} / \mathrm{l}\end{array}$ & $\begin{array}{l}\text { MAE since feed } \\
\text { start, } g / l\end{array}$ & $\begin{array}{l}\text { MAPE since inoculation, } \\
\%\end{array}$ & $\begin{array}{l}\text { MAPE } \\
\text { since feed } \\
\text { start, \% }\end{array}$ \\
\hline 1 & Escherichia coli & 15 & 1.04 & 1.04 & 5.7 & 5.7 \\
\hline 2 & & & 0.96 & 0.96 & 4.11 & 4.11 \\
\hline 3 & & & 1.38 & 1.38 & 5.61 & 5.61 \\
\hline 4 & & 12 & 1.45 & 1.6 & 5.3 & 4.91 \\
\hline 5 & & 7 & 1.65 & 2.3 & 7.26 & 5.34 \\
\hline 6 & & & 2.5 & 3.3 & 6.9 & 7 \\
\hline 7 & & & 1.54 & 2.13 & 8.67 & 6.28 \\
\hline 8 & & & 1.29 & 1.94 & 7.57 & 6.12 \\
\hline 9 & & & 1.52 & 2.54 & 7.12 & 7.96 \\
\hline 10 & & & 1.55 & 2.09 & 9.35 & 6 \\
\hline 11 & & & 1.87 & 2.7 & 10.75 & 6.6 \\
\hline 12 & & & 1.16 & 1.55 & 6.88 & 6.61 \\
\hline 13 & & & 0.97 & 1.22 & 9.03 & 6.89 \\
\hline 14 & & & 0.78 & 1.01 & 9.88 & 6.26 \\
\hline 15 & & & 1 & 1.22 & 8.92 & 6.99 \\
\hline 16 & & & 0.76 & 1 & 7.14 & 7.85 \\
\hline 17 & & & 0.74 & 0.9 & 8.51 & 5.69 \\
\hline 18 & & & 1.18 & 1.54 & 7.83 & 7.55 \\
\hline 19 & & & 0.8 & 1.07 & 5.83 & 6.30 \\
\hline 20 & Yeasts & 5 & 0.29 & 0.29 & 6.69 & 6.69 \\
\hline 21 & & & 0.66 & 0.66 & 7.28 & 7.28 \\
\hline
\end{tabular}



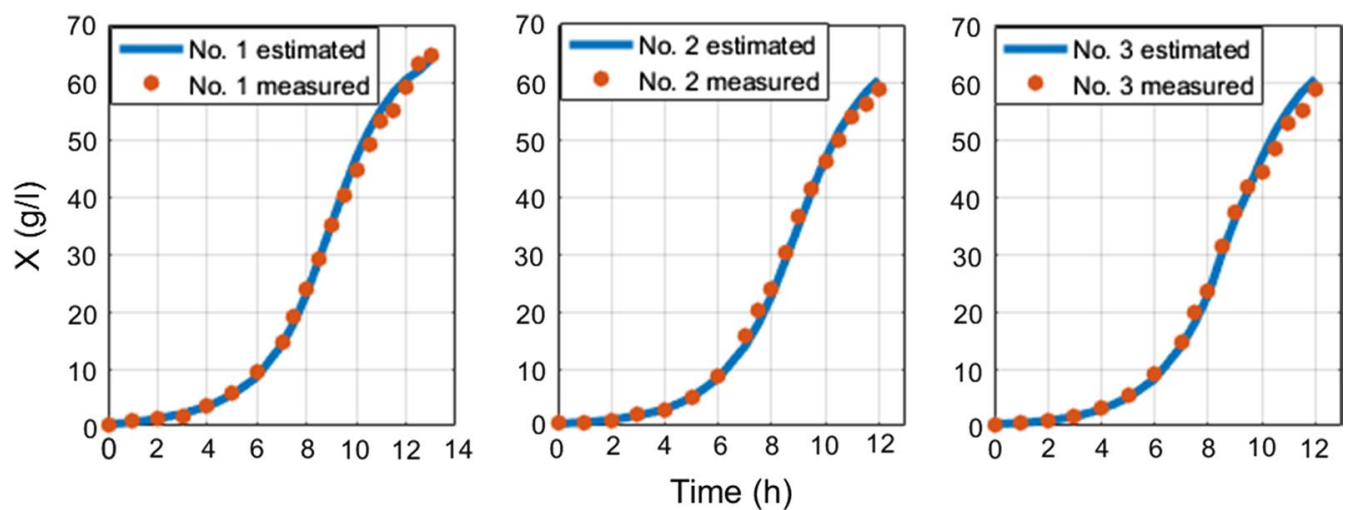

Fig. 8 Biomass concentration estimation result with recombinant E.coli BL21(DE3) pLysS strain at $15 \mathrm{~L}$ bioreactor

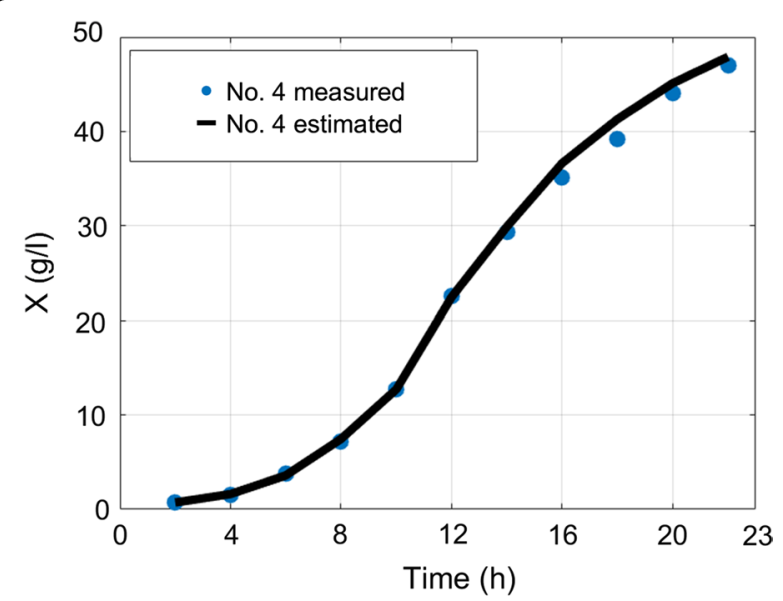

Fig. 9 Biomass concentration estimation result with recombinant $E$. coli BL21(DE3) pET9a-IdeS strain at 12 I bioreactor

optimization procedure were used. The speed of online estimation can be explained by the fact that the prediction value of biomass concentration estimate is calculated once during the whole estimation procedure. There is no updating performed for the predicted value of biomass. In the future, this optimization condition might be released though. The substrate feed was started from the beginning of cultivation process right after inoculation moment in the experiments \#1-\#3 and \#20-\#21, while for the rest of cultivations had their substrate feed started after 5-6 h since inoculations. The errors between off-line and on-line data mainly originate from offline measurements. Especially in \#5-\#19, because historically the accuracy of offline measurements was not of high priority during these experiments. Therefore, in the future the true ground truth of biomass concentration might testify that the approach suggested in this work has even higher overall precision than the one stated in above. All biomass state estimation results are shown at the Figs. 8 , 9, 10, 11, 12 .

\section{Conclusions}

The suggested biomass estimation's numeric approach using cumulative oxygen uptake rate signal showed no dependability on selection of the initial variable values for optimization procedures. This study assumed, by Pareto principle, that the proposed method is only dependent on stoichiometry parameters of the strain, i.e. the developed noninvasive biomass estimation procedure was made to not depend on both the manipulation with a specific growth rate variable and the selection of corresponding bioreactor parameters. The precision errors, since the bioprocess start, when inoculant was injected to a bioreactor, confirmed that the approach is relevant for online biomass state estimation. This included the lag and exponential growth phases for both E. coli and S. cerevisiae. The experimental investigation of E. coli and S. cerevisiae cultures showed that the estimation procedure is identical for both cultures. The overall average MAE of biomass estimation since inoculation is $1.1 \mathrm{~g} / \mathrm{l}$ and the overall average MAPE of biomass 

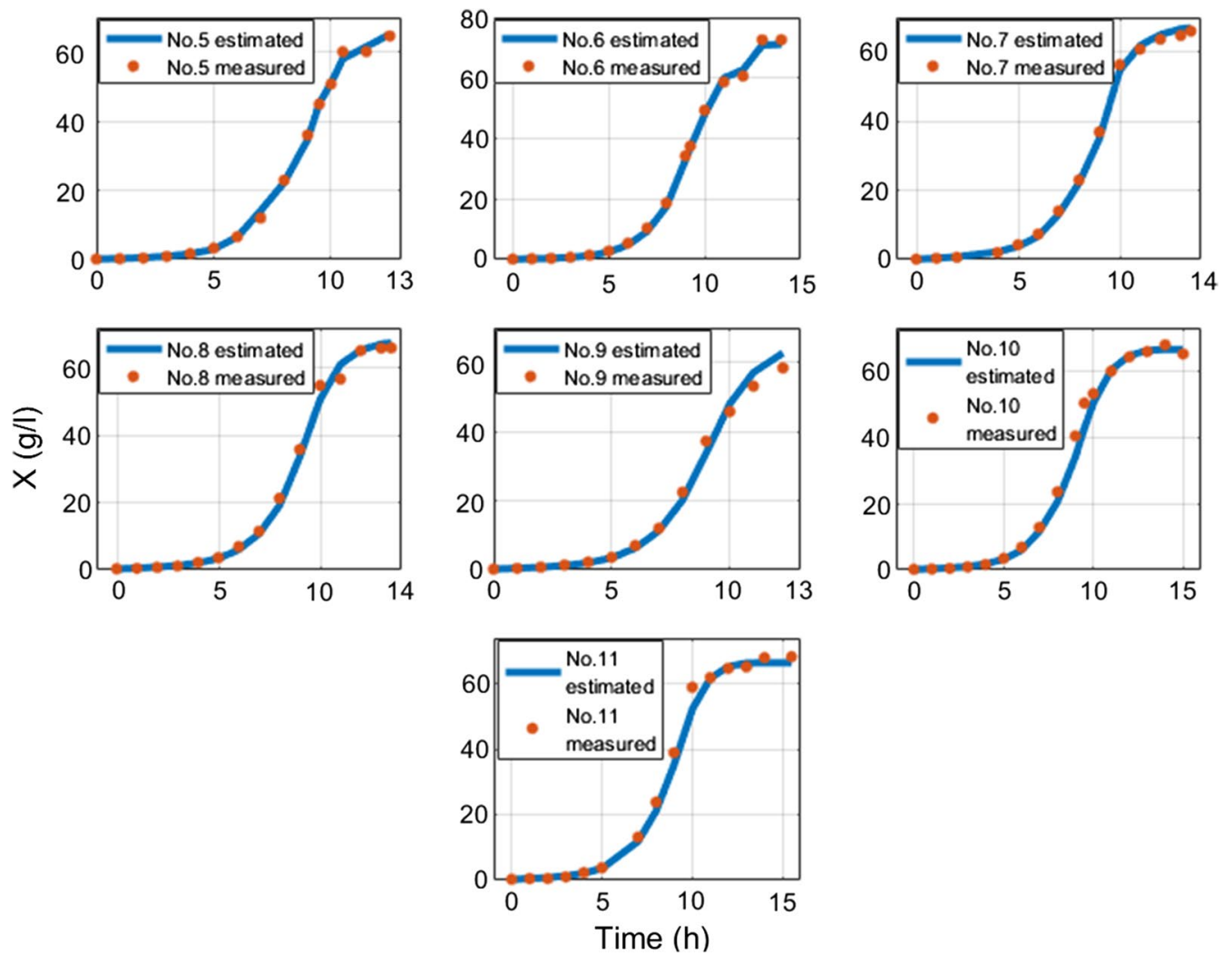

Fig. 10 Biomass concentration estimation result with recombinant E. coli BL21 (DE3) pET21-IFN-alfa-5 strain at $7 \mathrm{~L}$ bioreactor with dosed substrate feeding

estimation since inoculation is $7.28 \%$. RMSE value of $E$. coli cultivations before stationary phase, when DCW reaches $\sim 40 \mathrm{~g} / \mathrm{l}$ (to compare with results of other authors) with limited substrate feeding, is $1.07 \mathrm{~g} / \mathrm{l}$ and for cultivations with dosed substrate feeding is $1.2 \mathrm{~g} / \mathrm{l}$. These results show that this approach improves the precision achieved by other authors without compromising the simplicity of the implementation. Moreover, the suggested approach is a candidate method to be the microorganisms' culture invariant approach, it does not depend on any numeric initial optimization conditions, and it does not require any of bioreactor parameters. No numeric stability issues of convergence occurred during multiple performance tests. All this makes this approach a potential candidate for industrial tasks with adaptive feeding control or automatic inoculations when substrate feeding profile and bioreactor parameters are not provided.

Neither numeric artifacts nor abrupt worst-case scenarios were experienced during both offline and online analysis of 21 experiments, out of which 7 ones were carried out with dosed substrate feeding. The experiments executed in 5 l, 7 l, 121 and 151 bioreactor volumes. Feed start, inoculation, bioreactor medium, feeding limitation and other conditions varied with no manual control or adjustment. This encourages the use of such estimator in adaptive feedback control systems. Both online and offline estimations were tested on a single core CPU processing and each procedure took no more than $30 \mathrm{~ms}$ when overall 1-min interval data was sampled 

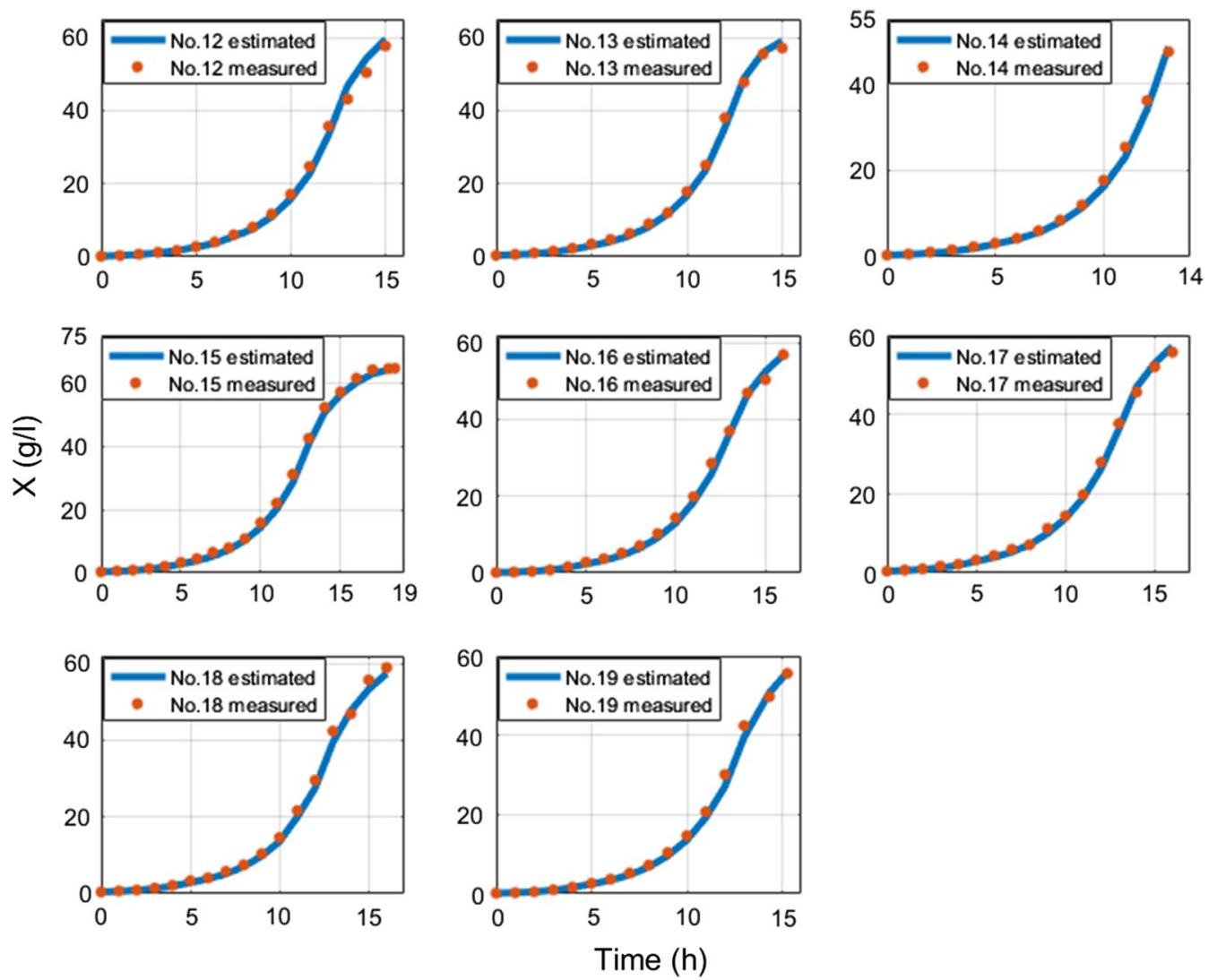

Fig. 11 Biomass concentration estimation result with recombinant E. coli BL21 (DE3) pET21-IFN-alfa-5 strain at $7 \mathrm{~L}$ bioreactor with limited substrate feeding

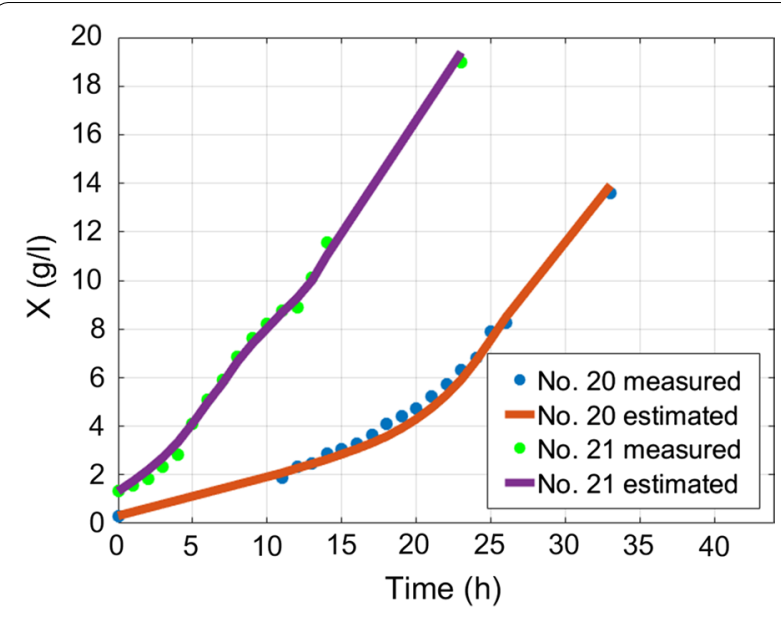

Fig. 12 Biomass concentration estimation result with Saccharomyces cerevisiae (no DY7221) strain at $5 \mathrm{~L}$ bioreactor

from cumulative oxygen uptake signal, which makes the approach of practical use too. Finally, this estimator does require a usage of regular industrial gas analysis equipment such as BlueSens etc.

\section{Acknowledgements}

We are grateful to professor Rimvydas Simutis (Kaunas University of Technology) for kindly providing the motivation and support that inspired and encouraged this publication.

\section{Authors' contributions}

$\mathrm{RU}$ and $\mathrm{AS}$ contributed to the preparation of the manuscript. All authors have read and approved the final manuscript. Conceptualization: $\mathrm{RU}$; methodology: $R U$; software: RU, AS; validation: RU, AS, DP; formal analysis: RU, AS; investigation: RU, AS, DP; writing —original draft preparation: AS, RU; writing — review and editing: RU, VG; supervision: VB, RU; project administration: RU, VB, VG; funding acquisition: VB, RU, VG. All authors read and approved the final manucript.

\section{Funding}

This research was funded by the European Regional Development Fund according to the supported activity "Research Projects Implemented by Worldclass Researcher Groups" under Measure No. 01.2.2-LMT-K-718.

\section{Availability of data and materials}

Some datasets used and analyzed during the current study are available from the corresponding author on reasonable request.

\section{Ethics approval and consent to participate}

Not applicable.

\section{Consent for publication}

Not applicable. 


\section{Competing interests}

The authors declare that they have no competing interests.

\section{Author details}

1 Department of Automation, Kaunas University of Technology, 51367 Kaunas, Lithuania. ${ }^{2}$ Biopharmaceutical Division of Centre for Innovative Medicine, 08406 Vilnius, Lithuania.

Received: 31 July 2019 Accepted: 23 October 2019

Published online: 05 November 2019

\section{References}

1. OPS Process Analytical Technology-(PAT) Initiative. https://www.fda. gov/regulatory-information/search-fda-guidance-documents/pat-frame work-innovative-pharmaceutical-development-manufacturing-and-quali ty-assurance. Accessed 31 Oct 2019

2. Goodwin GC. Predicting the performance of soft sensors as a route to low cost automation. Annu Rev Control. 2000;24:55-66. https://doi. org/10.1016/S1367-5788(00)90013-0.

3. Larroche C, Sanromán MÁ, Du G, Pandey A, editors. Current developments in biotechnology and bioengineering: bioprocesses, bioreactors and controls. Amsterdam: Elsevier; 2016.

4. Schaepe S, Kuprijanov A, Sieblist C, Jenzsch M, Simutis R, Lübbert A. Current advances in tools improving bioreactor performance. CBIOT. 2013;3:133-44. https://doi.org/10.2174/2211550102666131217235246.

5. Galvanauskas V, Volk N, Simutis R, Lübbert A. Design of recombinant protein production processes. Chem Eng Commun. 2004;191:732-48. https ://doi.org/10.1080/00986440490276056.

6. Simutis R, Lübbert A. Bioreactor control improves bioprocess performance. Biotechnol J. 2015;10:1115-30. https://doi.org/10.1002/ biot.201500016.

7. Bumelis VA. European Patent No. EP2532734A1; 2012. https://paten ts.google.com/patent/EP2532734A1. Accessed 31 Oct 2019.

8. Schaepe S, Kuprijanov A, Simutis R, Lübbert A. Avoiding overfeeding in high cell density fed-batch cultures of E. coli during the production of heterologous proteins. J Biotechnol. 2014;192:146-53. https://doi. org/10.1016/j.jbiotec.2014.09.002.

9. Rosenfeld E, Beauvoit B, Blondin B, Salmon J-M. Oxygen consumption by anaerobic Saccharomyces cerevisiae under enological conditions: effect on fermentation kinetics. Appl Environ Microbiol. 2003;69:113-21. https:// doi.org/10.1128/AEM.69.1.113-121.2003.

10. van Dijken JP, Weusthuis RA, Pronk JT. Kinetics of growth and sugar consumption in yeasts. Antonie Van Leeuwenhoek. 1993;63:343-52. https ://doi.org/10.1007/BF00871229.

11. Gnoth S, Kuprijanov A, Simutis R, Lübbert A. Simple adaptive $\mathrm{pH}$ control in bioreactors using gain-scheduling methods. Appl Microbiol Biotechnol. 2010;85:955-64. https://doi.org/10.1007/s00253-009-2114-5.

12. Mansano R, Godoy E, Porto A. The benefits of soft sensor and multi-rate control for the implementation of wireless networked control systems. Sensors. 2014;14:24441-61. https://doi.org/10.3390/s141224441.

13. Galvanauskas V, Simutis R, Levisauskas D, Repšyte J, Lübbert A. Comparison of state estimation techniques for biotechnological processes. In: 8th international conference on electrical and control technologies, ECT 2013; p. 70-5.

14. Linko $P$, Zhu Y. Neural network programming in bioprocess variable estimation and state prediction. J Biotechnol. 1991;21:253-69. https://doi. org/10.1016/0168-1656(91)90046-X.

15. Luedeking R, Piret EL. A kinetic study of the lactic acid fermentation. Batch process at controlled pH. Biotechnol Bioeng. 1959;1:393-412. https ://doi.org/10.1002/jbmte.390010406.

16. Simutis R, Galvanauskas V, Levisauskas D, Repsyte J, Vaitkus V. comparative study of intelligent soft-sensors for bioprocess state estimation. JOLST. 2013. https://doi.org/10.12720/jolst.1.3.163-167.

17. Unrean P. Bioprocess modelling for the design and optimization of lignocellulosic biomass fermentation. Bioresour Bioprocess. 2016;3:1. https:// doi.org/10.1186/s40643-015-0079-z.
18. Caramihai M, Severi I. Bioprocess modeling and control. In: Matovic MD, editor. Biomass now—sustainable growth and use. Rijeka: InTech; 2013. https://doi.org/10.5772/55362.

19. Gnoth S, Jenzsch M, Simutis R, Lübbert A. Process Analytical Technology (PAT): batch-to-batch reproducibility of fermentation processes by robust process operational design and control. J Biotechnol. 2007;132:180-6. https://doi.org/10.1016/j.jbiotec.2007.03.020.

20. Wechselberger $P$, Sagmeister $P$, Herwig C. Real-time estimation of biomass and specific growth rate in physiologically variable recombinant fed-batch processes. Bioprocess Biosyst Eng. 2013;36:1205-18. https:// doi.org/10.1007/s00449-012-0848-4.

21. Schubert J, Simutis R, Dors M, Havlik I, Lübbert A. Bioprocess optimization and control: application of hybrid modelling. J Biotechnol. 1994;35:51-68. https://doi.org/10.1016/0168-1656(94)90189-9.

22. Jenzsch M, Simutis R, Eisbrenner G, Stückrath I, Lübbert A. Estimation of biomass concentrations in fermentation processes for recombinant protein production. Bioprocess Biosyst Eng. 2006;29:19-27. https://doi. org/10.1007/s00449-006-0051-6.

23. Gnoth S, Jenzsch M, Simutis R, Lübbert A. Control of cultivation processes for recombinant protein production: a review. Bioprocess Biosyst Eng. 2008;31:21-39. https://doi.org/10.1007/s00449-007-0163-7.

24. Galvanauskas V, Simutis R, Lübbert A. Hybrid process models for process optimisation, monitoring and control. Bioprocess Biosyst Eng 2004;26:393-400. https://doi.org/10.1007/s00449-004-0385-x.

25. Aehle M, Simutis R, Lübbert A. Comparison of viable cell concentration estimation methods for a mammalian cell cultivation process. Cytotechnology. 2010;62:413-22. https://doi.org/10.1007/s10616-010-9291-z.

26. Petkov SB, Davis RA. On-line biomass estimation using a modified oxygen utilization rate. Bioprocess Eng. 1996;15:43-5. https://doi.org/10.1007/ BF00435527.

27. Barrigón JM, Ramon R, Rocha I, Valero F, Ferreira EC, Montesinos JL. State and specific growth estimation in heterologous protein production by Pichia pastoris. AIChE J. 2012;58:2966-79. https://doi.org/10.1002/ aic.12810.

28. Karim MN, Rivera SL. Artificial neural networks in bioprocess state estimation. Modern biochemical engineering. Berlin: Springer; 1992. p. 1-33. https://doi.org/10.1007/bfb0000703.

29. Caticha A. Entropic priors. In: AIP conference proceedings. jackson hole, Wyoming (USA): AIP; 2004. p. 371-80. https://doi.org/10.1063/1.1751380.

30. Gencaga D, Knuth K, Rossow W. A recipe for the estimation of information flow in a dynamical system. Entropy. 2015;17:438-70. https://doi. org/10.3390/e17010438.

31. Garcia-Ochoa F, Gomez E, Santos VE, Merchuk JC. Oxygen uptake rate in microbial processes: an overview. Biochem Eng J. 2010;49:289-307. https ://doi.org/10.1016/j.bej.2010.01.011.

32. Sivashanmugam A, Murray V, Cui C, Zhang Y, Wang J, Li Q. Practical protocols for production of very high yields of recombinant proteins using Escherichia coli. Protein Sci. 2009;18:936-48. https://doi.org/10.1002/ pro.102.

33. Shiloach J, Fass R. Growing E. coli to high cell density-a historical perspective on method development. Biotechnol Adv. 2005:23:345-57. https://doi.org/10.1016/j.biotechadv.2005.04.004.

34. Bohlin T. Practical grey-box process identification: theory and applications. London: Springer; 2006.

35. Schuler MM, Marison IW. Real-time monitoring and control of microbial bioprocesses with focus on the specific growth rate: current state and perspectives. Appl Microbiol Biotechnol. 2012;94:1469-82. https://doi. org/10.1007/s00253-012-4095-z

36. Swokowski EW. Calculus with analytic geometry. 2 d ed. Boston: Prindle, Weber \& Schmidt: 1979

37. Urniezius R, Galvanauskas V, Survyla A, Simutis R, Levisauskas D. From physics to bioengineering: microbial cultivation process design and feeding rate control based on relative entropy using nuisance time. Entropy. 2018;20:779. https://doi.org/10.3390/e20100779.

38. Giffin A, Urniezius R. The Kalman filter revisited using maximum relative entropy. Entropy. 2014;16:1047-69. https://doi.org/10.3390/e16021047.

39. Monod J. The growth of bacterial cultures. Annu Rev Microbiol. 1949;3:371-94. https://doi.org/10.1146/annurev.mi.03.100149.002103. 
40. Giffin A, Urniezius R. Simultaneous state and parameter estimation using maximum relative entropy with nonhomogenous differential equation constraints. Entropy. 2014;16:4974-91. https://doi.org/10.3390/e1609 4974.

41. Urniezius, R. Convex programming for semi-globally optimal resource allocation; 2016. p. 040002. https://doi.org/10.1063/1.4959056.

42. Willmott C, Matsuura K. Advantages of the mean absolute error (MAE) over the root mean square error (RMSE) in assessing average model performance. Clim Res. 2005;30:79-82. https://doi.org/10.3354/cr030079.
43. de Myttenaere A, Golden B, Le Grand B, Rossi F. Mean absolute percentage error for regression models. Neurocomputing. 2016;192:38-48. https ://doi.org/10.1016/j.neucom.2015.12.114.

\section{Publisher's Note}

Springer Nature remains neutral with regard to jurisdictional claims in published maps and institutional affiliations.
Ready to submit your research? Choose BMC and benefit from:

- fast, convenient online submission

- thorough peer review by experienced researchers in your field

- rapid publication on acceptance

- support for research data, including large and complex data types

- gold Open Access which fosters wider collaboration and increased citations

- maximum visibility for your research: over $100 \mathrm{M}$ website views per year

At BMC, research is always in progress.

Learn more biomedcentral.com/submissions 